\title{
A New Mechanism for Traffic Reduction the Service/Resource Discovery Protocol in Ad-Hoc Grid Network
}

\author{
Abdolsaeed Izadi $^{1}$, Alireza Sahab, Javad vahidi \\ Department of computer, Arak Branch, Islamic Azad University, Arak, Iran \\ Department of engineering, Lahijan Branch, Islamic Azad University, Lahijan, Iran \\ Department of Applied Mathematics, Iran University of Science and Technology, Behshahr, Iran \\ as.izadi@qmail.com, sahab@liau.ac.ir, j.vahidi@iust.ac.ir
}

\author{
Article history: \\ Received January 2013 \\ Accepted February 2013 \\ Available online February 2013
}

\begin{abstract}
In today's society the usage of Laptops, Cell phones, PDAs and the devices which have the ability of processing are increasing. This has led to innovating ideas which can raise the capacity of these devices which eventually resulted in the discovery of Selective Grids. The use of this capacity in various services made the discovery of Service possible as an important issue in this area. In recent years different solutions were proposed for Service and Source in Selector Grids. All of these solutions try to decrease the communication and processing's overloads in networks. This paper is an effort to propose a dynamic algorithm in which the selective grids can adopt themselves with their environment, in addition to falling the overloads down.
\end{abstract}

Keywords: Ad-hoc Grid, Service Discovery, Fault Tolerant.

\section{Introduction}

Nowadays, Grid computing is one of Discussions in computer science and it has been greatly focused on the IT industry. In fact Grid, combines computing resources, storage resources, and extensive network facilities. This cooperation can be used to access to powerful and extended computing services at any location that the grid network member is present. Grid technology has made possible the scientific collaboration that seemed impossible ,by creating a powerful, safe and high performance mechanism for discovery, access and optimizing remote resources. Moreover, Grid calculations provide users with storage services, display and analysis for using the distributed resources in a wide geographic range.

(MANET), wireless mobile ad-hoc network is a network that consists of some wireless hosts that can be mobile. In these Networks no predetermined infrastructure is used. Ad-hoc networks are used in many cases because their implementation is quick and easy and also they are independent of prefabricated structures.

\footnotetext{
${ }^{1}$ Corresponding author
} 
Ad-hoc Networks can be used in many personal applications such as Laptop connections to each other and their public applications such as general vehicles Communication, military applications, navy communications and emergency applications such as relief and rescue operations.[1]

The technological advancements in the Microprocessors and microcontrollers fields, sensors, and Wireless communications that they have been doing recently they have been combined with important practical applications and have led to a new model called ad-hoc Grid. An ad-hoc grid is a heterogeneous system that has not fixed infrastructure and all its components are mobile. Ad-hoc Grid is hierarchical of systems that they have various hardware, software and communications capabilities. Ad-Hoc grid environments allow users to share Processing resources, services and information.

Some of the issues needing a suitable solution in the Ad - Hoc grid include Resource and service discovery, energy consumption, Service quality, security and etc. Service discovery techniques in traditional Wired Grid environments are based on hierarchy methods that collect index servers or centralized directories of information on all services and available resources. In ad-hoc environments that there is not any predetermined organizing. The centralized mechanisms do not seem appropriate. Also it was shown that in wireless environment, the wireless channel quality changes according to time $[2,3]$ that this can affect routing algorithms and service discovery in Grids which are made in such networks.

Different approaches have been provided for service and resource discovery in wired traditional Grid environments that with the genesis of the new phenomena of the ad - hoc grid, some of them was trying to be implemented in the new environment. But because the wired environment and wireless environments in the ad - hoc grid are essentially different from each other, implementing of such a work may lead to a weak performance. Then many works have been done for service and resource discovery in wireless ad-hoc grids, any of these methods has its own particular communication and computational overhead that has a desired performance in certain conditions. But According to the dynamicity of the wireless environment, the condition changes and causes the proposed Static algorithms have acceptable performance for service and resource discovery at a particular time. In this article we offer such an algorithm for discovering the services and resources that can be able to adapt itself to changing environmental conditions in wireless ad-hoc environment, such as dense and adapting their despairs and holding their overhead at a reasonable level. At last we evaluate it with simulating the proposed algorithm and comparing it with other studies have done in this field .

\section{Related Work}

Flexibility and minimum user manipulation are Essential characteristics of communication networks in future. These networks must be employed easily and they should be easy to re-configure when New hardware and software capabilities is added to them.

One of the essential characteristics of such networks it then. Self-configurable in service discovery. The service discovery provides the ability for Systems in networks to discover network services via their characteristics automatically and also they announce their own abilities to Networks. For this purpose, several industrial and organization Consortium( that some of them Overlap with together SLP) formed for standardizing different service discovery protocols such as SLP ${ }^{2}$ Protocol from IETF [4] ,sun company's Jini

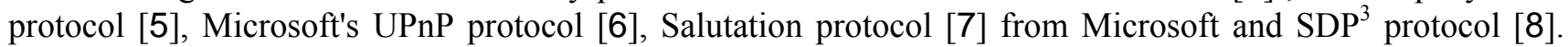
The key concept in wireless ad-hoc grid is configured automatically but for it standardization has not been performed any effort yet. Now, we introduce several proposed service and resource discovery methods and several solutions for wired grid infrastructure that are based on P2P distributed mechanisms [9]. The main problem in the ad - hoc grid is that the nodes are not fixed and they can be mobile, secondly, then they are faced with energy restrictions therefore the discovery Time must be short, that others should be able to give the service requests to them until Server nodes are available.

\footnotetext{
${ }^{2}$ Service Location Protocol

${ }^{3}$ Universal Plug and Play
} 
Service discovery mechanisms in the ad - hoc grid are highly primitive. At MANET There are two manners that through them devices can exchange their information: a) queries b) announcements Questions, including the types of devices that Send requests to the Network and other Devices that inform needed information to these devices in network and Client devices use them. Many of MANET applications uses a combination of queries and announcements. Publishing area of Questions and announcements and also periodic policy and announcements saving is such as important parameters that Determine performance and accuracy of discovery declarations remember the policy period and notified or the efficiency and accuracy of Service discovery in these networks.

In certain cases of mobile ad-hoc grid, the method declaration for the announcement is not sufficient. In these networks one of Information is the resource availability that it is possible these resources such as CPU load and available memory have Significant changes at short periods of time. The Traditional methods that discussed earlier such as Salutation and the Jini focus on a centralized directory, who upon it the services have registered or Discovered. Information of existing services will be focused in special Directory s. Rout and announcement of services are based on multicasting and service Registrations is based on uncasing. In general, centralized discovery directory has a good Infrastructure for the Internet and wireless networks, but such architecture is not suitable for MANET that is dynamic in nature. In addition in the directory there are Routing bottlenecks and although, this architecture can supply additional directory for scalability problem, but the cost of construction will be increased by increasing the Directory numbers.

In [10] has offered a method for service discovery in Grids. In fact they offered a middleware architecture that called MoGrid. MoGrid for source discovery uses a method based on the question. Have offered to discover the source uses. In dichotomy an applicant node sends The IREQ requesting messages to other nodes in mobile ad-hoc that query on them for resources providing. IREQ messages will be repeated in each intermediate node to form a controlled flood that is limited to max-Hop parameter. A Demand Node sends a IREQ message to $\mathrm{T}$ node (that is a common intermediate node and the $\mathrm{T}$ node sending this message to all Its neighbors). This process Would continue until arrive to the max Hop parameter.

The hybrid service discovery method is one of the methods that offered later. This method is a mixed of protocols based on questions and announcements [11-13]. These protocols try to prevent undeserved increasing and On the other hand, they reduce the large delay of it algorithms that were based on questions.

In [11] each server node has a Constant area, where in this area it declares its Services. In first Each demand node that want a service, it search about the service in itself area and If it does not find the desired service, therefore it sends the service request in broadcast form to out of itself area and In this case operate similar to Reactive protocol.

\section{Proposed Method}

In this section, we introduce the offered algorithm, DHSD. The DHSD ${ }^{4}$ method provides the possibility of multi hop, automatic and dynamic service discovery in MANET networks. Also DHSD supplies the conditions for mobile nodes to find their desired requests quickly and it does not require that all nodes introduce themselves to entire networks periodically or all of the nodes ask about server about Presence or absence of the service. That means only the servers are aware of its service. DHSD is an algorithm without any distributed directory and it allows mobile nodes react against certain links and connections, in addition to DHSD changes, topology, density and network size. The DHSD is an algorithm without a cycle.

One of the special features of DHSD is the using of a serial number (SN) related to service agents that retain for each service. In DHSD each node in its announcement area, keep the number of neighbors, which regarding fix Radius of sending the nodes, this task is a special expression of the density. The SN of each service agent will be made by itself, Thereby it will be saved in whole services information. In ad-hoc Grid, server nodes are not providing just a specific service, but several different services and each demand node may require Several Services simultaneously. DHSD define several types of messages. The service discovery

\footnotetext{
${ }^{4}$ Dynamic Hybrid Service Discovery
} 
message that the demand node creates it and uses it for service agent discovery. The reply message that is finally created by severing node. The error message that is produced as a result of using a route that does not exist at present. The announcement message that will be used for a service announcement in its own area. The demand node uses the IP headers for packets and the source IP address as the name of the demand service. We regulate the SR publication domain with the TTL field in the IP header. In this protocol each node has a table as table service. This offered protocol is responsible for MANET networks with tens of thousands of nodes and also hundreds of Service. Such a DHSD algorithm can tolerate different levels of nodes mobility. It does not consider any Overload or any add-on security package for this proposed method and We have assumed that all the nodes are safe and there is not any destructive intrusive node in the network and security considerations can be considered as a research field in further. In previously mentioned service discovery protocols, there is a compromise between the overhead and the service discovery delay. In The reactive or active protocols, the overhead is often limited and The discover delay is vast and in passive or based on announcement protocols, the overhead is usually vast but the discovery delay is low and the hybrid protocols have been designed in order to achieve to the optimized point in this Compromise. But hybrid protocols in MANET networks have their own particle Problems, since the topology and network variation will cause every default in the The design of the protocols for service discovery in the networks result in the performance of the algorithm going further from optimized point. Therefore designing such a Protocol that could adapt itself to different network conditions, seems to be necessary.

In this study we try to prepare a hybrid protocol that can be able to adapt itself to different network conditions. In this study, the nodes that give the address of a server node called directory and the nodes that provide the service called service [14]. The node that prepares a specific service, must announce its service to its own area. There are two states for not know about the network, it considers its area radius a default value, otherwise it calculates its area radius based on node density around it. The service announcement to area nodes of each node has special overhead that has a direct relationship with the density and the radius of node area. The following figure shows this obviously.
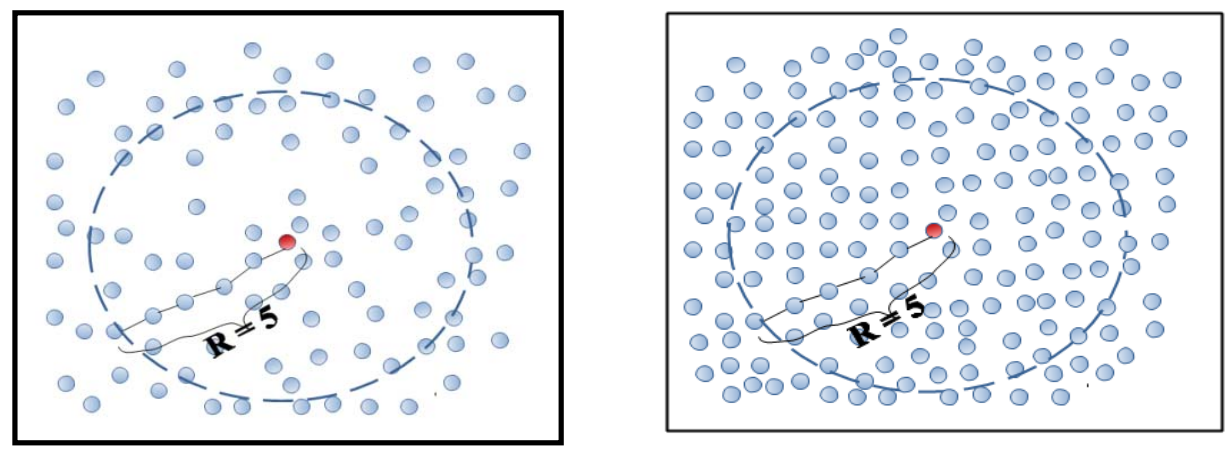

Figure1: Effect of Density on the Number of Nodes in Neighborhood of Fixed Radius

As we have seen in the figure, the greater the density is, the more the number of nodes will be, so in a constant radius the announcement overload will be greater. In our algorithm, the area radius is dynamic and will be determined based on several parameters. In dynamic ad-hoc network, regarding changes of network 
conditions, some of the network parameters can be changed and also it may be necessary to change the area radius. A sample pseudo-code for this work is shown in the figure at the below.

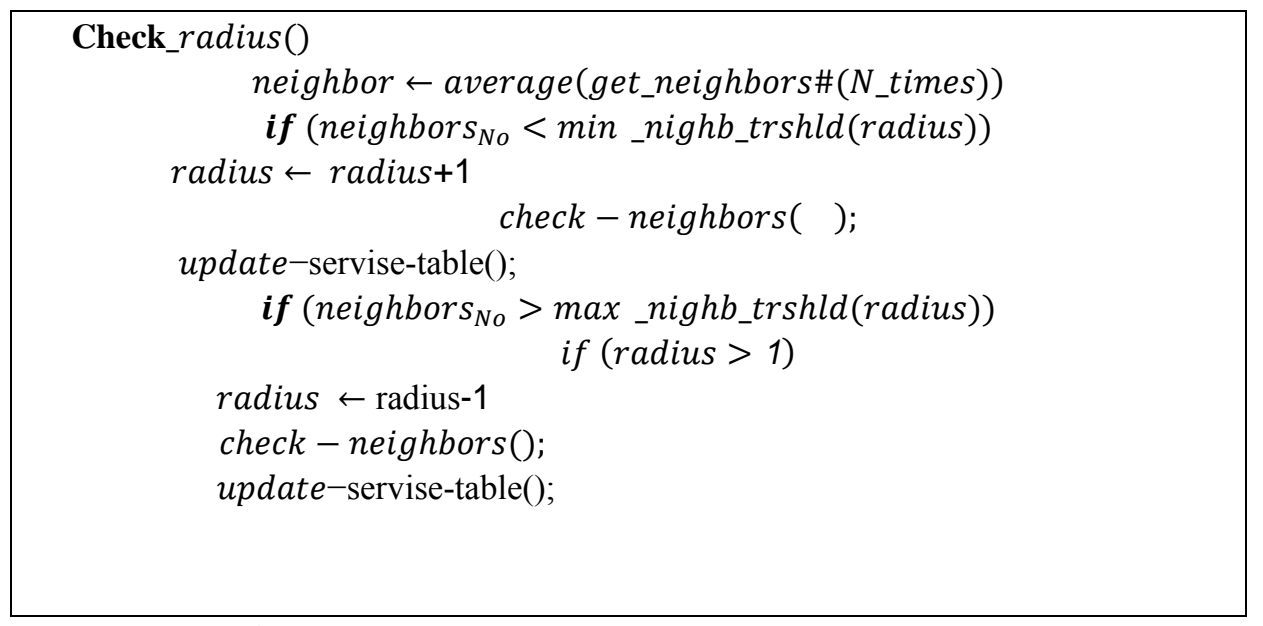

Figure2: Change the Radius based on Change the number of node

In the first line number of neighbors of each node will be determined. If it is less than a threshold value, the radius will increase and the service table will be updated. Also if the radius is greater than a threshold value, the radius will be decreased and the service table will be updated again. Determination of the suitable threshold value is dependant on network and the environment that this algorithm is implemented in it. For example, if such a sensor network is used that the amount of energy is Vital and delay can be tolerated, the threshold value will be lesser since the announcement overload is lower and the network nodes energy consumption is lower. But in Networks wich are so sensitive to the delay, the threshold value must be considered much greater in order to more numbers of nodes operate as directory agent and the protocol will be closer to deactivate protocols and energy consumption will be much higher. Here our purpose is Wireless ad- hoc network grid in wich there are devices with medium energy such as PDA, laptop and Mobile (that it play the client role). Energy Consumption is essential for us and on the other hand, the delay should be kept at an accepted rate. In this protocol, each network node has a table called neighborhood Table. in this table be keeps the immediate information of Neighboring nodes with a certain number of hops and also all nodes in its node area, next hop to reach that node and the number of hops to reach the node are kept in it. By using this table each node can be aware of the number of nodes in its area so it can set the radius of its announcement area. Also nodes have a table called service table. This table includes a description of the services and the service providers. Content of rows in this table includes servers' services in its node area, requests for services and their replies which have been provided for demandant node by the route, enter the service into their service table and keep it until its expiration .[15]

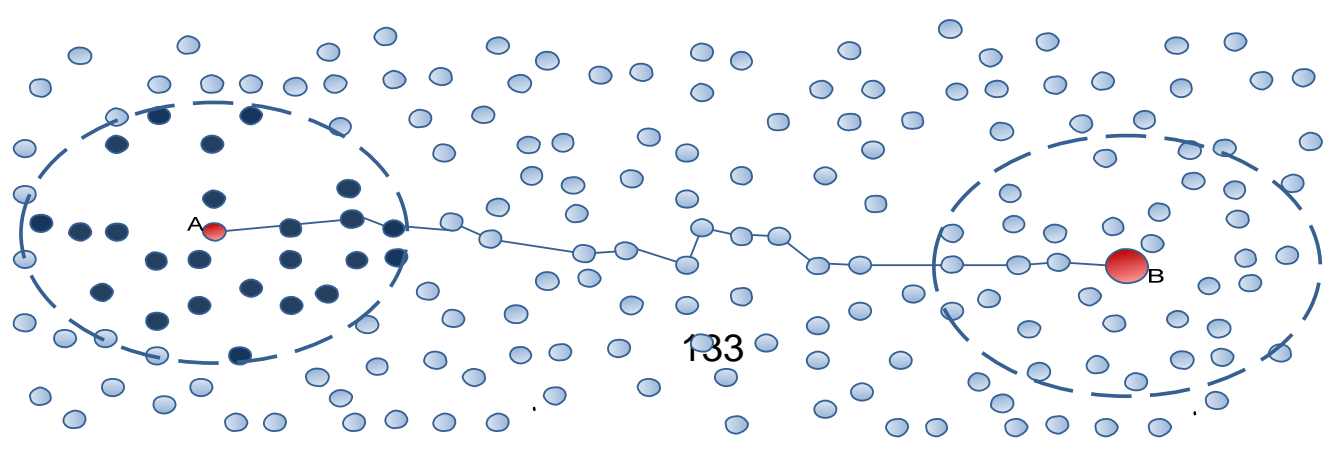


Figure 3: Client service and respond service to requests for service

By assuming that the node a node wants a service that is offered by node B. node A Searches its service table, whereas it cannot find the desired service, it send a service request to the boundary nodes. with broadcasting this request, boundary nodes search it on neighborhood areas. When service request reaches to node B or to one of nodes in node B area, they supply service respond message and send it to demandant

node, based on finding the service in their tables. When one of neighbors of node B in its area or a node out of this area that knows service provider receives the packet,it sends the service request to node $\mathrm{B}$ in uni-casting way. If B receive the request while didn't receive it previously, B Gives back this request packet to demandant in a reverse route. Then the demandant sends its data to service provider in the route that response is reached to client service, on the other hand service provider offers its services in this way and gives back service results to demandant, if needed. All nodes in this route keep the service on their table until expiration time of the service and operate as a directory for it.

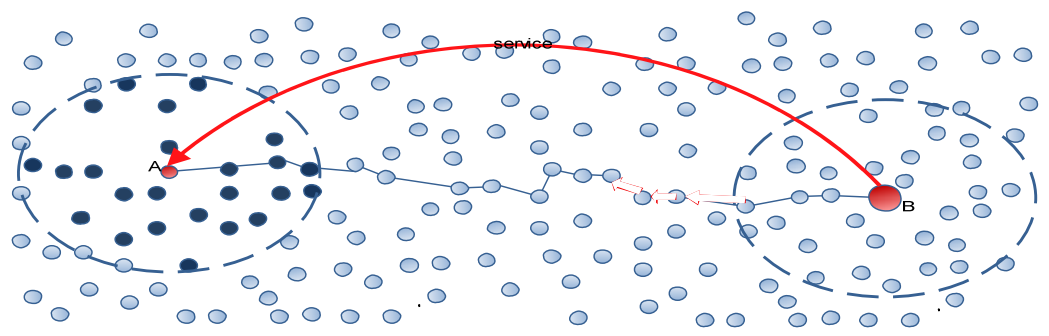

Figure 4: Response Service Path in Grid Computing

Thus as it lasts from inauguration of the network, the timeline for finding the service will be shorter.Because there will be routes of services that for finding a specific service, it's not necessary to reach the server node area. Also as the varieties of services are fewer, again during the time, timeline by finding the service will be shorter. Because more nodes will be aware of server nodes.Now in this scenario, with considering due to nodes mobility or turning on of new nodes and entering other nodes into network, the density of the environment of node B will be greater. Therefore, node B will be aware of an increasing number of nodes in its area with periodic messages then Reduces its radius. The following figure shows this process.

\section{Experiments}

We implement our offered method (DHSD) by prowler ${ }^{5}$ simulator that Written via Matlab software. We model the network environment as $300 \mathrm{~m} * 300 \mathrm{~m}$ that in it A set of nodes dispersed randomly and with Uniform distribution function. The first performance Criterion that we do our evaluation via it; it is the number of announcement messages that protocols use them to register Service in directory s or other nodes. As regard the simulation environment is constant, As a result, increasing the number of nodes in this space, leads

\footnotetext{
${ }^{5}$ Probabilistic Wireless Network Simulator
} 
to increase the density of the network. As you know, whatever the number of messages is less, therefore less overhead Is imposed to network, lower and thereupon energy consumption be lower Than before. But generally the overhead Reducing to announcement messages is not without cost and it enhance the detection delay.

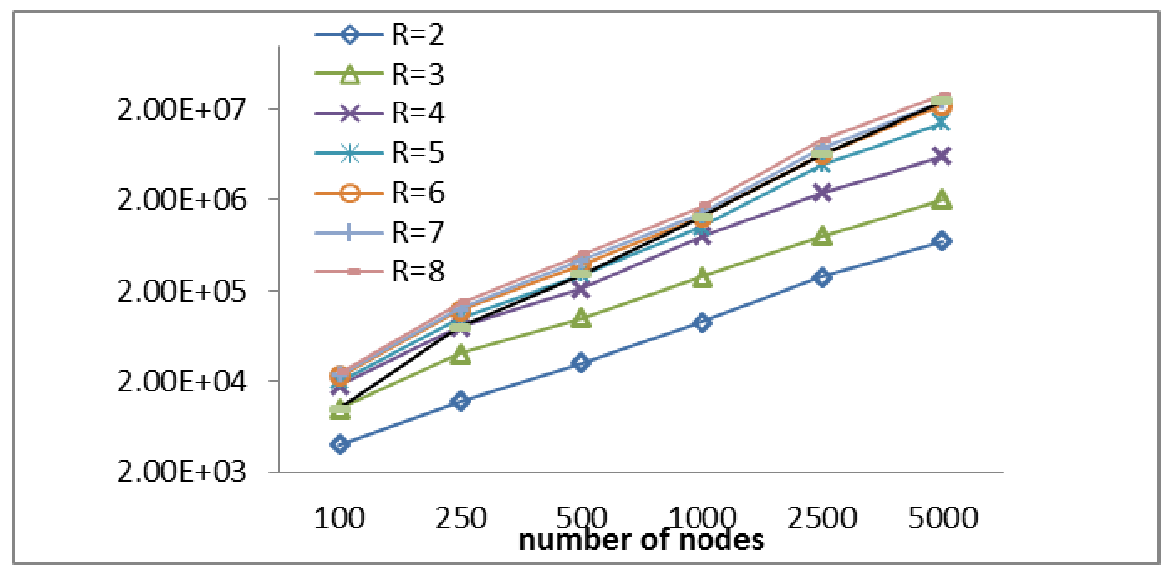

Figure 5: Number of advertisement messages based on the number of nodes in the environment

As we see in Figure 5, the minimum number of announcement messages is related to hybrid protocols with a radius that valued 2. Due to Different density and less number of nodes, and regard to small radius of Nodes area in this case, the result is not unexpected and it is natural. The number of announcement messages increase Due to the announcement number of nodes.

This increase is for two reasons. First, the number of nodes be increased and the number of areas increases consequently. Then each node has more nodes in its radius with value 2 . Whatever The area radius be increased, thus the number of announcement messages will be increased and more Hybrid protocol get away from service discovery protocols that are based on request and be neared to service discovery protocols that are based on announcements.

As we see, in first the proposed protocol considers the medium rate for number of announcement messages and whatever be increased the number of nodes thus be increased number of announcement messages be increased and be neared to Hybrid protocols with greater transmission radius. This work has been done based on policy for achieve the maximum percent of Service discovery.

If we consider a threshold value for discovery percent and regulate it to a low amount, our protocol in cases with many nodes move to side Hybrid diagrams with less radius that has low threshold value. In design of this protocol what we consider was establishing a reliable protocol with high percent of service discovery protocol. 


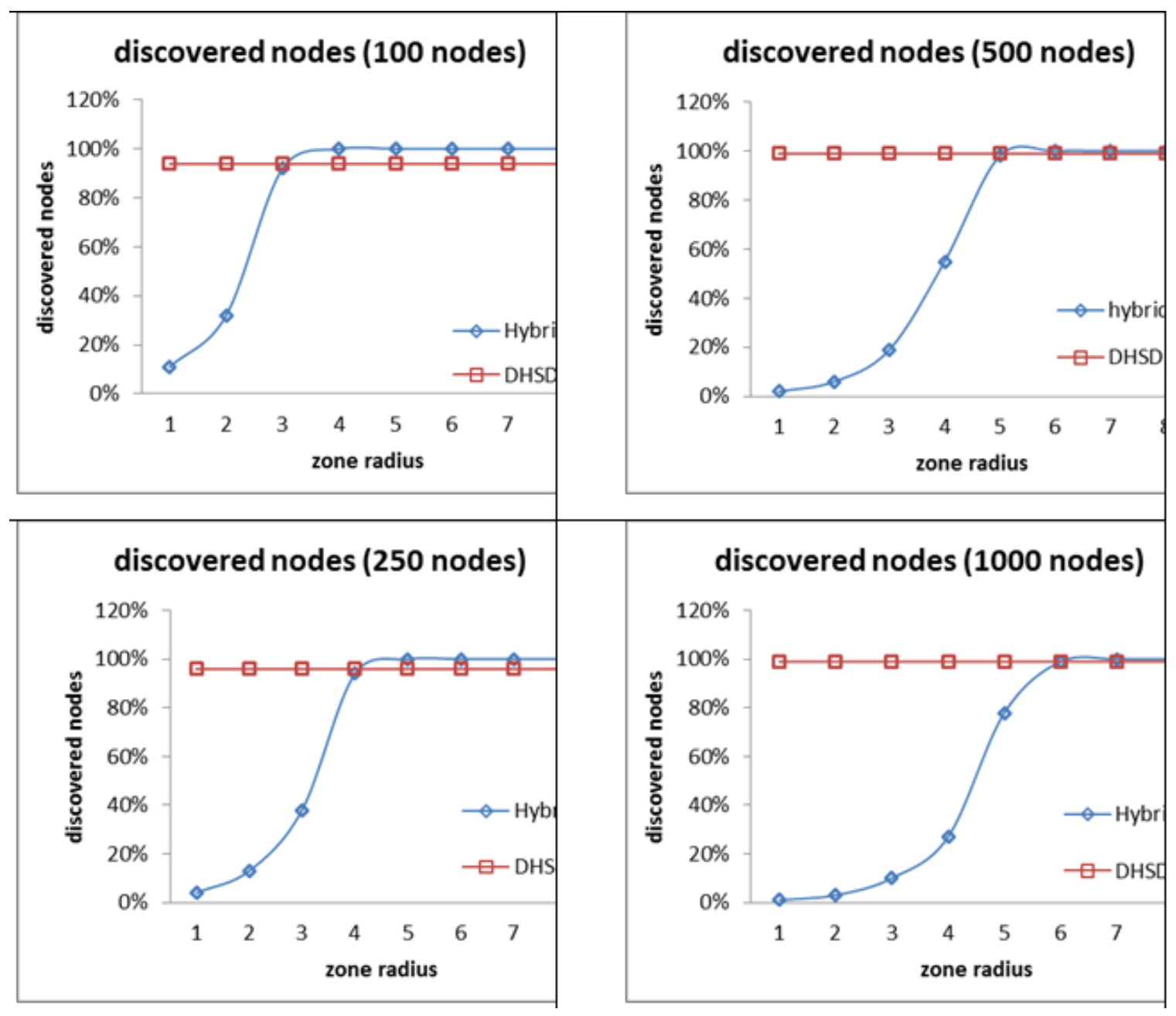

Figure 6: the chart of percent on discovered nodes at radius area in the number of variety nodes

In the above diagram the horizontal axis is the radius for hybrid algorithms. Because our algorithm has not a constant Radius and determine its radius regard to the it's environment. Thus the curve of DHSD in every six scenarios is a line in fact and shows a fixed point. As we see in diagrams regard to number of nodes since a specific radius the hybrid method is better and gives a higher Percent service discovery.

But if we regard to before diagram, we find that the hybrid method on large radius will be operate Similar to based on announcement method and made much more overhead than our method. Thus DHSD in balancing between overhead and Percent service discovery since a specific radius will be trend to lower overhead and refers it then Percent service discovery and don't accept consumption all network recourses for slight increase in Percent service discovery. Whatever The number of nodes increases, the Hybrid method arrive to greater Percent service discovery in large radius and this cause is increasing the number hops and packets loss in routs because all requests Do not reach to destination. The DHSD Algorithm prefers the possibility to arrive to a high rate Service discovery with a reasonable overhead. 


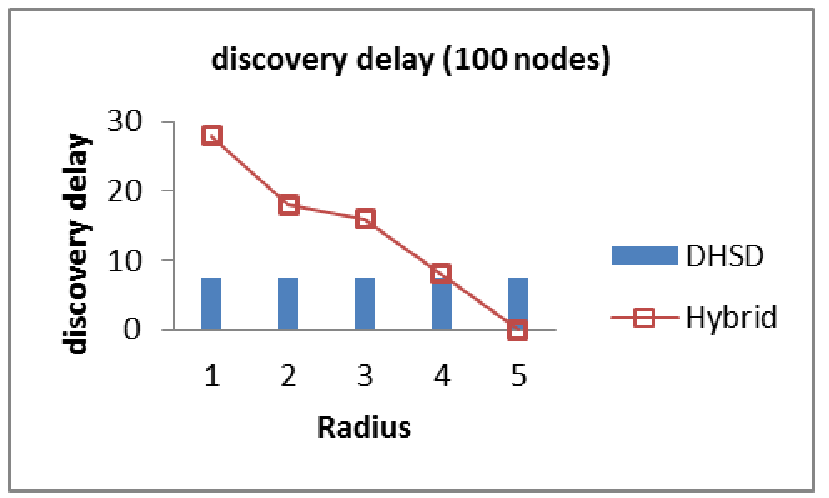

Figure 7: Discovery Delay based on Radius Area of the two algorithm hybrid and DHSD

When a node search a service until it finds the service is some associated with delays that named discovery delay. The above diagram shows the discovery delay based on area radius in as scenario with 100 nodes. Bar diagram are equal because Such as

We have mentioned, Cannot be adjusted a constant radius for this algorithm. As we see, for Most of the radiuses, our protocol has less delay then hybrid protocol. However, this work had specific calculation costs for us. Our algorithm is suitable for Networks that they will not delay service discovery become higher than expected level, and special success percent be expected. However, we are not oblivious to overheads and we have tried to keep the overhead at acceptable level.

\section{Conclusions for future works}

In this paper a method has been preprade that apply a combination of two methods and the algorithm has been designed so that is capable to Reacts against changes to their environment and adopts itself to variable conditions of wireless environment. That each server node announce its services in a specific range and this range is variable depending on the wireless environment conditions. In this present work, a Criterion has been applied for coordinating with the environment that is discovery overhead. This coordination can be done based on Multi Criterion. For example, it can use a function of two criteria,the delay and overhead. moreover, in each network several routes can be considered for discovering the service. For example, nodes in the network boundary or nodes in the networkdiameter can be considered as directory nodes .

Thus, the server nodes register their services in network diameter and send registration requests to network boundary or network diameter and the demandant nodes send their requests to directory bands.

\section{References}

[1] S. Ghasempour, S.H. Kamali, M. Hedayati, R. Shakerian, A Priority Scheduler Based QoS for Dynamic Source Routing Protocol Using Fuzzy Logic in Mobile Ad-Hoc Network, 3, 329 - 338, (2011). 
[2] M.D.Yarvis, et.al .Real world experiences with an interactive ad hoc sensor network. In Proceedings of Parallel Processing Workshops, (2002), P.P. 143-151.

[3] R. Stutz. Performance analysis and optimization of a $2.4 \mathrm{GHz}$ multihop, wireless self-configurable network. MS Paper, Univ of California, Berkeley, (2008)

[4] E. Guttman, et.al, Service Location Protocol, Version 2, Vinca Corporation, RFC, (1999).

[5] http://www.jini.org, The Jini Community, (2000)

[6] wwwupnporg/resources/UPnPbkgndhtm, Microsoft Corporation, Universal Plug and Play, (1999).

[7] wwwsalutationorg/specordrhtm, Consortium Salutation Architecture Specification, (1999).

[8] E.A. Gryazin, Service discovery protocol, Bluetooth Specification Version 1.1, Part E, (2001).

[9] M. Dyrna, Peer2peer Network Service Discovery for Ad hoc Networks, Seminar on Ad Hoc

Networking, Technical University of Munich, (2004).

[10] A.A. Gomes, et.al, DICHOTOMY: A Resource Discovery and Scheduling Protocol for Multihop Ad Hoc Mobile Grid, in Proceedings of CCGRID '07 Proceedings of the Seventh IEEE International Symposium on Cluster Computing and the Grid, (2007), Washington, DC, USA.

[11] R.Vozmediano, A hybrid mechanism for resource/service discovery in ad-hoc grids. Future Generation Computer Systems, 7, 717-727 (2009).

[12] T. Abdullah, et al, Hybrid Resource Discovery Mechanism in Ad Hoc Grid Using Structured Overlay Architecture of Computing Systems, in proceedings of ARCS '09 22nd International Conference on Architecture of Computing Systems, (2009); Springer-Verlag Berlin, Heidelberg.

[13] A. Parichehreh, et al, Reliable Hybrid Service Discovery Protocol in Mobile Ad-Hoc Grid, in Proceedings of $10^{\text {th }}$ IEEE Computer and Information Technology (2010); Washington, DC, USA.

[14] ] R. Marin-Perianu, P. Hartel, H. Scholten, A Classification of Service Discovery Protocols, Technical report TR-CTIT-05-25, Centre for Telematics and Information Technology, University of Twente, The Netherlands, June (2005).

[15] E. Meshkova, J. Riihijrvi, M. Petrova, P. Mhnen, A Survey on Resource Discovery Mechanisms PeerPeer and Service Discovery Frameworks, Computer Networks, 4, 2097-2128 (2008). 